\title{
Interpretation of ageostrophic winds and implications for jet stream maintenance
}

Article

Published Version

Blackburn, M. (1985) Interpretation of ageostrophic winds and implications for jet stream maintenance. Journal of the Atmospheric Sciences, 42 (23). pp. 2604-2620. ISSN 15200469 doi: https://doi.org/10.1175/15200469(1985)042<2604:IOAWAI>2.0.CO;2 Available at https://centaur.reading.ac.uk/34992/

It is advisable to refer to the publisher's version if you intend to cite from the work. See Guidance on citing.

Published version at: http://dx.doi.org/10.1175/1520-0469(1985)042<2604:IOAWAl>2.0.CO;2

To link to this article DOI: http://dx.doi.org/10.1175/1520-

0469(1985)042<2604:IOAWAI>2.0.CO;2

Publisher: American Meteorological Society

All outputs in CentAUR are protected by Intellectual Property Rights law, including copyright law. Copyright and IPR is retained by the creators or other copyright holders. Terms and conditions for use of this material are defined in the End User Agreement.

\section{www.reading.ac.uk/centaur}

\section{CentAUR}


Central Archive at the University of Reading

Reading's research outputs online 


\title{
Interpretation of Ageostrophic Winds and Implications for Jet Stream Maintenatice
}

\author{
MICHAEL BLACKBURN \\ Department of Meteorology, University of Reading, Reading RG6 2A4. England
}

(Manuscript received 17 August 1984, in final form 18 June 1985)

\begin{abstract}
The use of ageostrophic flow to infer the presence of vertical circulations in the entrances and exits of the climatological jet streams is questioned.

Problems of interpretation arise because of the use of different definitions of geostrophy in theoretical studies and in analyses of atmospheric data. The nature and role of the ageostrophic flow based on constant and variable Coriolis parameter definitions of geostrophy vary. In the latter the geostrophic divergence cannot be neglected, so the vertical motion is not associated solely with the ageostrophic flow.

Evidence is presented suggesting that ageostrophic flow in the climatological jet streams is primarily determined by the kinematic requirements of wave retrogression rather than by a forcing process. These requirements are largely met by the rotational flow, with the divergent circulations present being geostrophically forced, and so playing a secondary, restoring role.
\end{abstract}

\section{Introduction}

Historically there have been two definitions of geostrophy in use in geophysical fluid dynamics. What may be called the "synoptician's geostrophy" is based on a balance between Coriolis and horizontal pressure gradient forces and thus uses the local value of the Coriolis parameter. Denoting this as type-1 geostrophy (or geostrophy-1), the balance in isobaric coordinates is $f \mathbf{v}_{g^{1}}=\mathbf{k} \times \nabla \phi$, where the symbols have their usual meanings. In contrast, the "theoretician's geostrophy" has been defined mathematically as the zero order balance in a Rossby number expansion of the equations of motion (see, e.g., Phillips, 1963). The analysis is generally for horizontal scales small relative to the Earth's radius, so variations in the Coriolis parameter are neglected to zero order. This yields what will be denoted type- 0 geostrophy (or geostrophy -0 ), $f_{0} \mathbf{V}_{g 0}=\mathbf{k}$ $X \nabla \phi$, in which a constant value of the Coriolis parameter is used.

To zero order in the Rossby number expansion the two definitions are equivalent, and geostrophy-0 is an approximation to the balance of forces that is the basis of geostrophy-1. However, it will be shown that the first order balance and the ageostrophic flow remaining after removing the zero order balance have different interpretations in the two definitions. For this reason the nomenclature 'geostrophy-0' and 'geostrophy-1' appears useful in differentiating between the two. Geostrophy- 0 is chosen as a mnemonic referring to the constant $f_{0}$ definition. The geostrophic- 0 wind is nondivergent, but the divergence of the geostrophic-1 flow will be found to be a nonnegligible part of the total horizontal divergence, so it is not valid to infer the vertical motion in the atmosphere merely from the ageostrophic-1 flow.

The work of Blackmon et al. (1977) and Hoskins et al. (1983) has helped to resolve the question of how the climatological jet streams are maintained, whether by fluxes associated with the transient eddies or by the time-mean flow itself. Both studies show transient eddies to be important in the center and exit of the jets, leaving the accelerations in the entrance region to be provided by the time-mean flow. Together with other indicators, Blackmon et al. used the presence of timemean meridional ageostrophic-1 flow in both jet entrance and exit regions to infer the presence of vertical meridional circulations there. The entrance circulation is inferred to be thermally direct with rising motion on the equatorward side, and so the kinetic energy (KE) of the jet is maintained from a thermal energy source. This paper questions the direct inference of these vertical circulations from the pattern of ageostrophic-1 wind alone. Of course none of this answers the question of how jet streams are related to the centers of tropical convection, where large-scale ascent is located. This and the more general question of the positioning of the major jet streams are still largely unanswered.

The two types of geostrophy are analyzed in Section 2 and their differences illustrated in Section 3, where ageostrophic flow in a nondivergent Rossby mode is studied. The analysis is extended to a more realistic model situation in Section 4, where the energetics of localized jets directly associated with tropical heating and in a remotely forced Rossby wavetrain are considered. The Northern Hemisphere winter jet streams for $1982 / 83$ are studied in Section 5, after which conclusions are presented. 


\section{Theoretical considerations}

In order to study the role of the ageostrophic flow in the two definitions of geostrophy it is necessary to use a consistent set of momentum and vorticity equations. This is not possible using a classical linear balance system that retains the variation of the Coriolis parameter $\left[\nabla^{2} \phi=\nabla \cdot(f \nabla \psi)\right]$ because the energetically consistent vorticity and divergence (balance) equations each imply a different approximation to the momentum equation. The analysis here will therefore be of the first-order equations in a Rossby number expansion of the hydrostatic primitive equations, as in the analysis of Phillips (1963). The Rossby wave example in Section 3 uses a linear balance system in the context of horizontally nondivergent flow.

The equations of motion governing adiabatic and inviscid flow on a midlatitude $\beta$-plane may be written in isobaric coordinates as

$$
\begin{gathered}
D \mathbf{V} / D t=-\left(f_{0}+\beta y\right) \mathbf{k} \times \mathbf{V}-\nabla \phi \\
\nabla \cdot \mathbf{V}+\partial \omega / \partial p=0 \\
D T / D t-\kappa \omega T / p=0 \\
\partial \phi / \partial p=-R T / p,
\end{gathered}
$$

where the symbols have their usual meanings. The horizontal divergence in (2) will be denoted $D=\nabla \cdot \mathrm{V}$.

Consider scales of motion for which the Rossby number Ro and the ratio of the horizontal length scale, $L$, to the Earth's radius, $a$, are both small and of the same order, so that $L / a \sim$ Ro $\sim 1 / 6$. Let $V$ be the velocity scale.

Following Phillips' (1963), a strict scale analysis yields geostrophy- 0 as the zero order balance in a Rossby number expansion, $f_{0} \mathbf{V}_{g 0}=\mathbf{k} \times \nabla \phi$, which is a horizontally nondivergent flow. In contrast, geostrophy-1 is defined by $f \mathbf{V}_{g 1}=\mathbf{k} \times \nabla \phi$ and has divergence $D_{g 1}=-\beta v_{g 1} / f$. Since $D_{g 1} \sim \mathrm{O}(V / a)=\mathrm{O}(\operatorname{Ro} V / L)$, this divergence of the geostrophic-1 wind is $O(R o)$ compared with the geostrophic-1 vorticity, so the two types of geostrophy are equivalent to zero order in Ro.

In order to study the roles of the two types of ageostrophic flow, each geostrophic balance will be subtracted from the momentum equation (1) in turn, and only terms of first order in Ro retained.

Subtracting the type- 0 geostrophic balance gives the first order momentum equation

$$
\left(\partial / \partial t+\mathbf{V}_{g 0} \cdot \nabla\right) \mathbf{V}_{g 0}=-f_{0} \mathbf{k} \times \mathbf{V}_{a 0}-\beta y \mathbf{k} \times \mathbf{V}_{g 0},
$$

where the ageostrophic-0 wind $V_{a 0}=V-V_{g 0}$ is $O(R o)$ compared with the geostrophic- 0 wind. The acceleration of the geostrophic- 0 flow is not simply determined by the ageostrophic- 0 flow and an extra term appears involving the geostrophic- 0 flow itself. This arises because the ageostrophic- 0 flow is not a measure of the local imbalance between the mass and wind fields that is the source of accelerations in the full equation (1). The vorticity equation derived from (5) is

$$
\left(\partial / \partial t+\mathbf{V}_{g 0} \cdot \nabla\right)\left(\zeta_{g 0}+f\right)=-f_{0} D_{a 0},
$$

where $D_{a 0}$ is the divergence of the ageostrophic- 0 wind. Since this is equal to the total divergence, the righthand side reduces to the usual stretching term and the vorticity balance is

$$
\left(\partial / \partial t+\nabla_{g 0} \cdot \nabla\right)\left(\zeta_{g 0}+f\right)=f_{0} \partial \omega / \partial p .
$$

The vertical motion is associated entirely with the ageostrophic- 0 flow, so the ageostrophic- 0 wind field can be used to imply the vertical motion.

Subtracting the type-1 geostrophic balance from (1) and retaining only terms of first order in Ro yields

$$
\left(\partial / \partial t+\mathbf{V}_{g 1} \cdot \nabla\right) \mathbf{V}_{g 1}=-f_{0} \mathbf{k} \times \mathbf{V}_{a 1} .
$$

Here the ageostrophic-1 flow is a measure of the local imbalance between the mass and wind fields and so determines the acceleration. This is readily visualized by rotating the ageostrophic-1 wind vector clockwise by $\pi / 2$ in the Northern Hemisphere. It is notable that there is no variation of Coriolis parameter explicit in (7). The vorticity equation derived from it is

$$
\left(\partial / \partial t+V_{g 1} \cdot \nabla\right) \zeta_{g 1}=-f_{0} D_{a 1}-\zeta_{g 1} D_{g 1},
$$

which does not explicitly contain the advection of planetary vorticity. This can be introduced via the definition of geostrophic-1 divergence, since $-f D_{g 1}$ $=\mathbf{V}_{g 1} \cdot \nabla f$. Then the vorticity balance is

$$
\left(\partial / \partial t+\mathbf{V}_{g 1} \cdot \nabla\right)\left(\zeta_{g 1}+f\right)=-f_{0} D-\zeta_{g 1} D_{g 1}-\beta y D_{g 1} .
$$

Thus the variation of Coriolis parameter arises in a different way in the two first-order equation sets. For geostrophy- 0 it is retained explicitly on the right-hand side of the momentum equation (5), whereas for geostrophy-1 it enters solely through the divergence of the geostrophic-1 wind when this is differentiated.

Scale analysis reveals the last two terms of $(8)$ to be

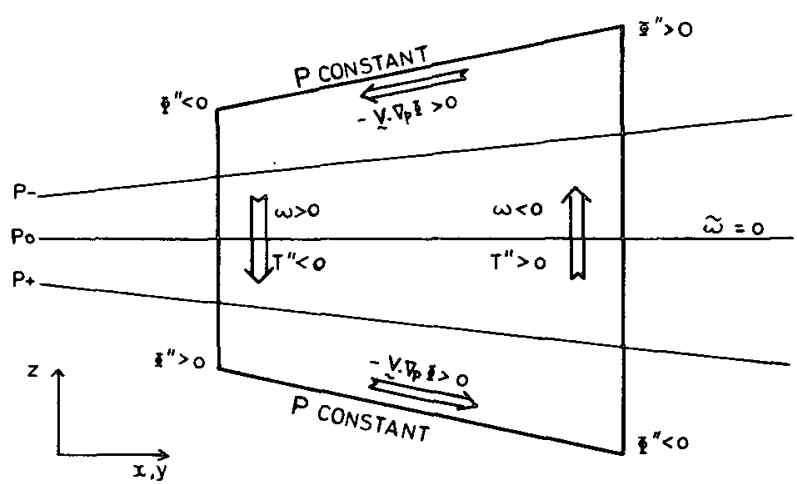

FIG. 1. Idealized flow in a thermally direct circulation that accomplishes a net conversion of thermal to kinetic energy. The KE generation occurs at upper and lower levels where the divergent wind is directed down the gradient of geopotential. Thermal energy changes occur at midlevels in the vertical branches. (") denotes an average over an isobaric surface and ()$^{\prime \prime}$ a local departure from that average. 
$\mathrm{O}(\mathrm{Ro})$ relative to the advection and main stretching term $-f_{0} D$, so the first order vorticity equations for geostrophy-0 and geostrophy- 1 are only equivalent to $\mathrm{O}(\mathrm{Ro})$. Also, a Rossby number expansion of the vorticity equation derived from the full momentum equation using geostrophy-1 would yield (6), but with $V_{g 1}$ replacing $\mathbf{V}_{g 0}$ throughout, which is only consistent with (7) and (8) to $O(R o)$. This is perhaps a reason for the widespread use of geostrophy- 0 in theoretical studies. See Eliassen (1984) for a discussion of this point.

It has been noted that the divergence of the geostrophic-1 flow is $\mathrm{O}(\mathrm{Ro} V / L)$, and it is of interest to compare this with the order of the total horizontal divergence. The fact that the zero order flow in the Rossby number expansion is nondivergent immediately reveals that the total divergence is also $\mathrm{O}(\operatorname{Ro} V / L)$ since it is

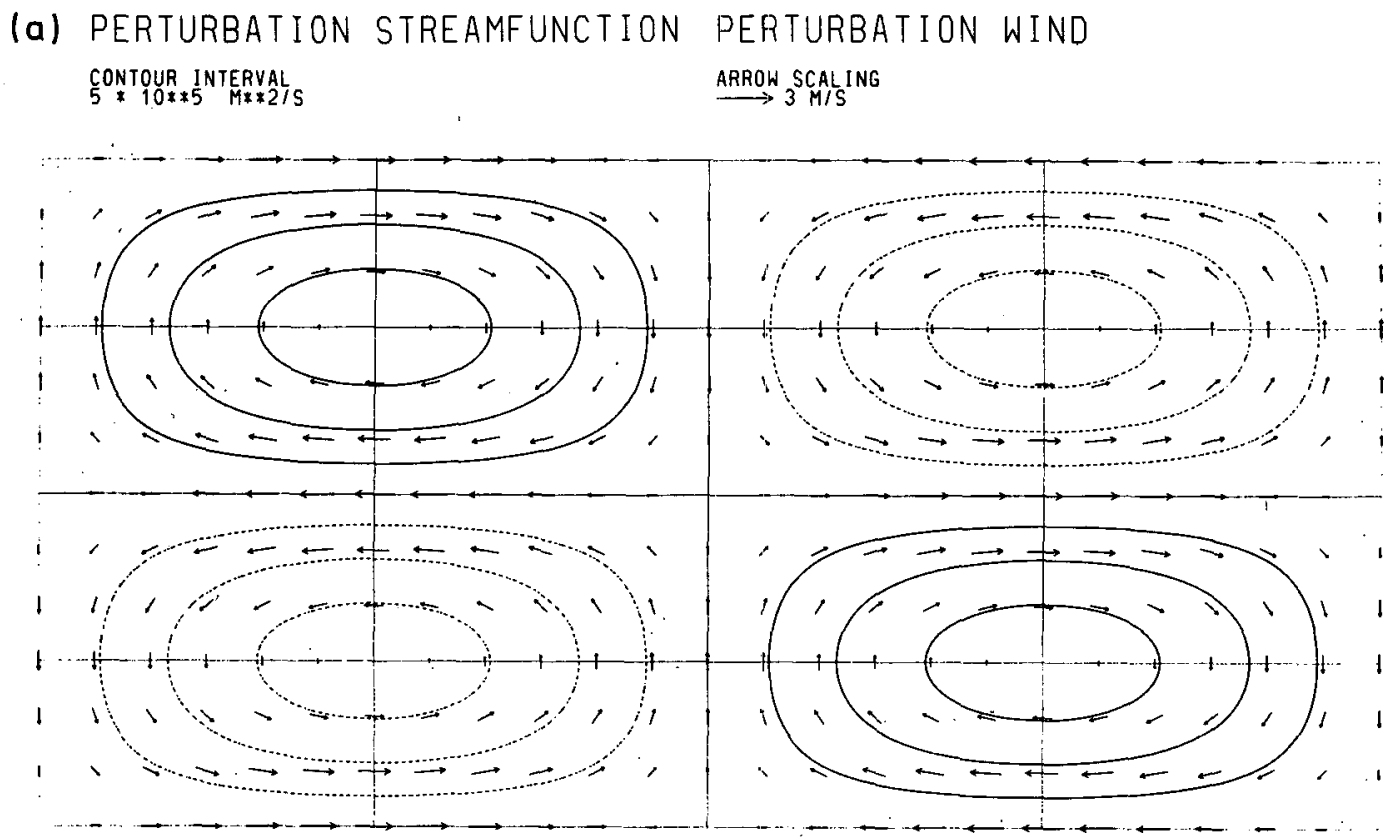

(b) TOTAL STREAMFUNCTION $\underset{5 * 10 * * 6}{\text { CONTOUR INTERVAL }}$ M**2/S

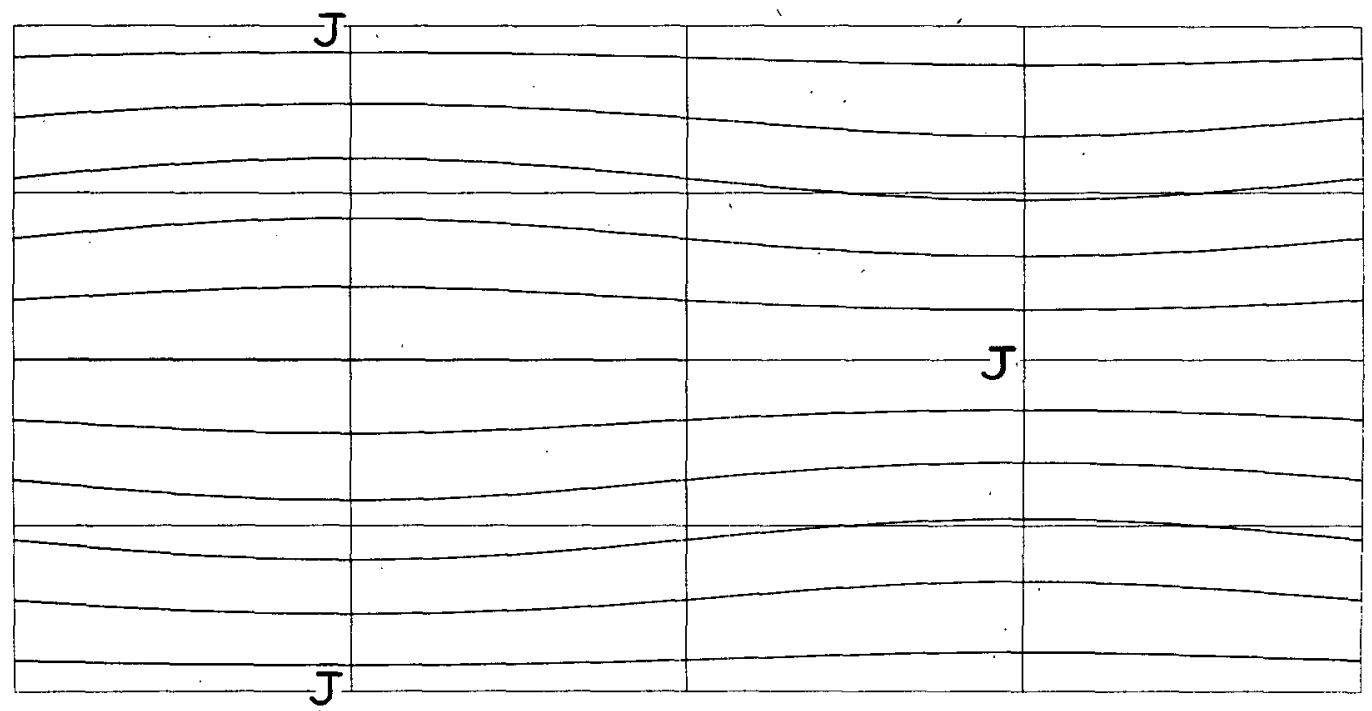

FiG. 2. Streamfunction for a free, nondivergent Rossby mode, with a uniform zonal flow $\bar{u}$ as a basic state. (a) Perturbation streamfunction and wind, and (b) total streamfunction, using $\tilde{u}=10 \mathrm{~ms}^{-1}$ and a wave amplitude of 2 $\mathrm{ms}^{-1}$. The total flow resembles a series of jetlike regions, the centers of which are denoted " $J$ ". 
merely the divergence of the first order flow $\mathbf{V}_{a 0}$, which is $\mathrm{O}(\mathrm{RoV})$. This result is also obtained from a scale analysis of the thermodynamic equation (3), again for Ro $\sim L / a \sim 1 / 6$, as in Phillips (1963). Further, the advection of planetary vorticity entered (8) only through the geostrophic-1 divergence, since $-f D_{g 1}$ $=\mathrm{V}_{g 1} \cdot \nabla f$. This term and the stretching $-f D$ form the Sverdrup balance in a linear analysis with zero basic state flow.

Thus the divergence of the geostrophic-1 flow is of the same order as the total horizontal divergence, for the midlatitude scales of motion considered here. It is therefore not valid to deduce the vertical motion from the divergence of the ageostrophic-1 flow alone.

The identification of vertical motion with the ageostrophic flow, valid only for geostrophy- 0 , has all too readily been retained in diagnostic studies of atmospheric data based on the variable- $f$ definition, geostrophy-1. For example, Blackmon et al. (1977) found meridional ageostrophic- 1 flow at upper levels in the entrances and exits of the climatological jet streams and interpreted it as the upper branches of vertical circulations in the meridional plane there. The scale analysis here suggests this to be invalid.

In a discussion of the quasi-geostrophic omega equation, Hoskins et al. (1978) have shown that the geostrophic-0 flow is in thermal wind balance but that changes in that flow tend to destroy the balance. The ageostrophic-0 and vertical circulation is geostrophically forced, being necessary to maintain the thermal wind balance of the evolving geostrophic- 0 flow. In the $f$-plane case, the vertical motion is conveniently visualized in terms of the " $Q$-vector". On a $\beta$-plane the diagnosis of vertical motion can be approached using either type of geostrophy. The form of the circulation equation obtained using geostrophy- 1 favors this formulation, despite the vertical motion not being entirely associated with the ageostrophic-1 flow. This is discussed in Appendix A.

The ageostrophic flow also enters the KE equation, and so is involved in the conversion from thermal energy. Subtracting either type of geostrophy from the full momentum equation (1) and forming the equation for the $\mathrm{KE}(k)$ of the horizontal motion yields

$$
D k / D t=-\mathbf{V} \cdot \nabla \phi=-\mathbf{V}_{a} \cdot \nabla \phi .
$$

The local generation of $\mathrm{KE}$ thus depends on the component of flow across the geopotential contours in an isobaric surface, whereas the enthalpy, $C_{p} T$ in (3), changes in flow through isobaric surfaces at a rate $R \omega T$ / $p$. The Helmholtz partitioning helps to clarify the link between these separate processes. Defining the rotational and divergent wind components to be $\mathbf{V}_{\psi}$ and $V_{\chi}$, respectively, the KE source term becomes $-\mathbf{V} \cdot \nabla \phi$ $=-\mathbf{V}_{\psi} \cdot \nabla \phi-\mathbf{V}_{x} \cdot \nabla \phi$. Pearce (1974) discusses these terms, calling them the barotropic and baroclinic sources, respectively.
For purely rotational motion there is still a local source of $\mathrm{KE}$ and air parcels slide up and down isobaric surfaces in closed circulations $(\omega=0)$, converting their energy between gravitational potential and kinetic forms. This component of the KE source integrates to zero over a closed region of an isobaric surface (if the normal components of both $\mathbf{V}_{\psi}$ and $\mathbf{V}_{\chi}$ are zero on the boundary) and so cannot provide a net source of $\mathrm{KE}$ to balance frictional dissipation. For this reason it will be called the "neutral" component.

In contrast, it is only the divergent motion, $\mathbf{V}_{x}$, which can provide a net generation in a closed domain to balance frictional dissipation of KE. This entails a net conversion from thermal energy since

$$
-\nabla_{x} \cdot \nabla \phi=-\widehat{R \omega T / p}
$$

over the domain, where $(\neg)$ denotes an average over mass. The KE generation and enthalpy changes still occur by different processes locally: their organization in a thermally direct circulation is indicated schematically in Fig. 1.

This behavior of the KE balance is in marked contrast to the vorticity balance, (6) or the zero-order component of (8), where the local source term depends only on the divergent wind via the stretching. The "neutral" component of the $\mathrm{KE}$ balance is viewed as a purely advective phenomenon.

\section{Ageostrophic flow in a nondivergent Rossby mode}

In order to illustrate the advantages and disadvantages of the two types of ageostrophic flow, a free nondivergent Rossby mode is now studied. The usual analysis of this mode is in terms of vorticity and streamfunction. Here the geopotential height field will be found and the flow partitioned into geostrophic and ageostrophic components for both type- 0 and type- 1 geostrophy.

Consider inviscid and horizontally nondivergent flow on a $\beta$-plane. Linear perturbations, ( $)^{\prime}$, of a constant zonal flow $\bar{u}$ that is in geostrophic balance are governed by

$$
\begin{aligned}
\partial u^{\prime} / \partial t+\bar{u} \partial u^{\prime} / \partial x & =\left(f_{0}+\beta y\right) v^{\prime}-\partial \phi^{\prime} / \partial x \\
\partial v^{\prime} / \partial t+\bar{u} \partial v^{\prime} / \partial x & =-\left(f_{0}+\beta y\right) u^{\prime}-\partial \phi^{\prime} / \partial y \\
\partial u^{\prime} / \partial x+\partial v^{\prime} / \partial y & =0 .
\end{aligned}
$$

Defining vorticity $\zeta$ and streamfunction $\psi$ in the usual way, Rossby and collaborators (1939) showed that

$$
(\partial / \partial t+\bar{u} \partial / \partial x) \nabla^{2} \psi^{\prime}+\beta \partial \psi^{\prime} / \partial x=0,
$$

which expresses conservation of absolute vorticity $\left(\zeta^{\prime}\right.$ $+f$ ). Solutions that are periodic in both $x$ and $y$ are plane waves that obey the Rossby dispersion relation, and so retrogress relative to the basic state flow. In order to visualize the wave structure, two particular 
(a) PERTURBATION GEOPOTENTIAL AGEOSTROPHIC-O WIND CONTOUR INTERVAL ARROW SCALING

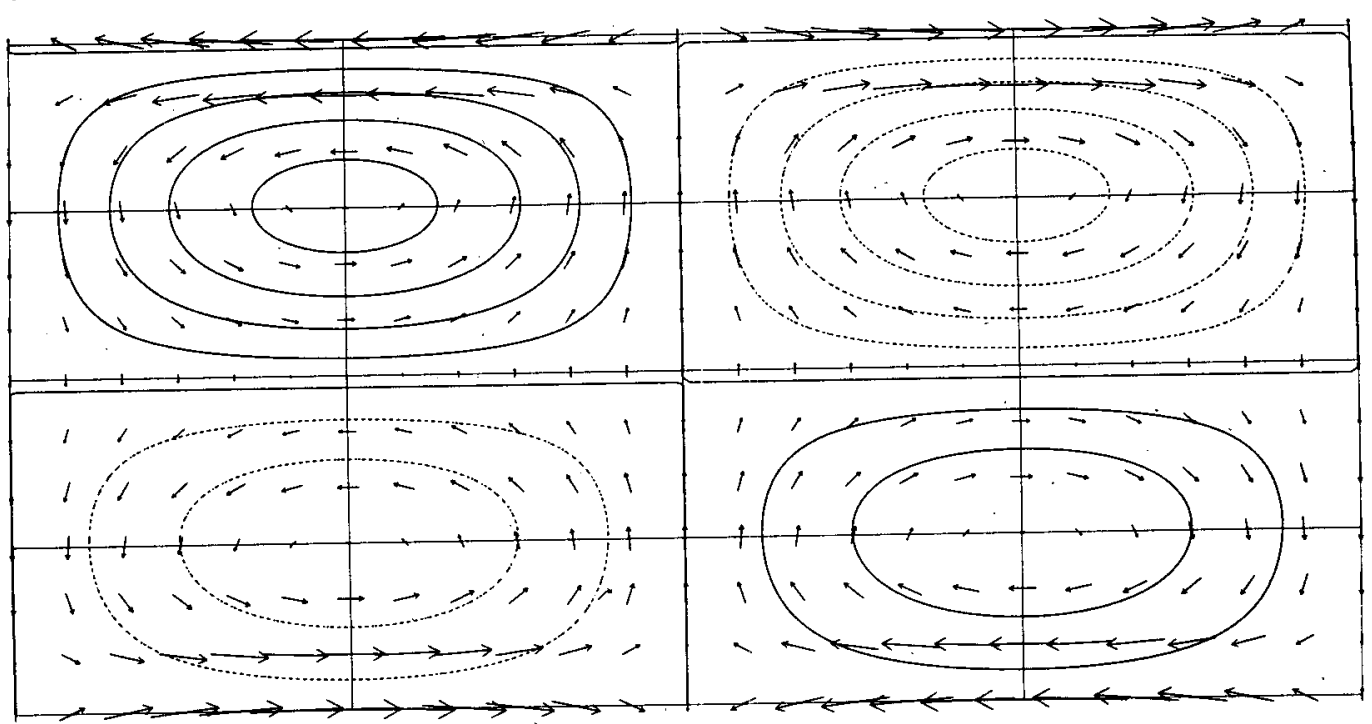

(b) TOTAL GEOPOTENTIAL AGEOSTROPHIC-0 WIND CONTOUR INTERVAL
500 J/KG $\stackrel{\text { ARROW SCALING }}{\longrightarrow} 0$ S

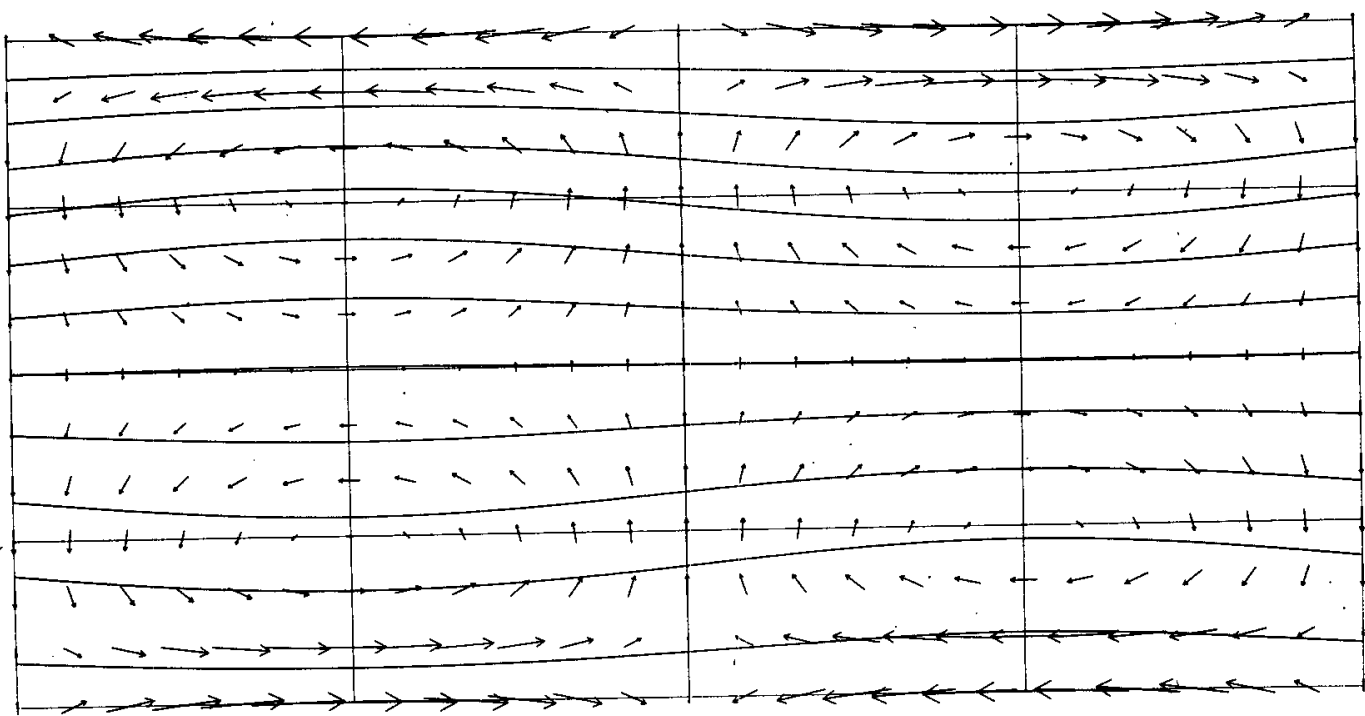

Fig. 3. Ageostrophic- 0 wind vectors for the Rossby mode of Fig. 2 based on the $f_{0}$-definition of geostrophy, together with the (a) perturbation, and (b) total geopotential. Since the geostrophic- 0 wind is nondivergent the ageostrophic- 0 wind is similarly nondivergent. (c) KE generation rate, depicting accelerations of air passing eastwards through the jet regions. Note that the accelerations cannot be visualized by rotating the ageostrophic- 0 wind vectors through $90^{\circ}$ using this type of geostrophy.

solutions are added to give a circular or elliptical disturbance. See Appendix B for details and for the form of the solutions for the various fields.

Figure $2 \mathrm{a}$ shows the perturbation streamfunction and winds in the wave, and Fig. $2 b$ the total flow including $\bar{u}$. There are regions of enhanced and depleted westerly wind and, since these retrogress relative to the basic state flow, air must accelerate as it passes through them from west to east. An ageostrophic wind component is necessary to provide these accelerations. 


\section{(c) TOTAL-KE GENERATION CONTOUR INIERVAL}

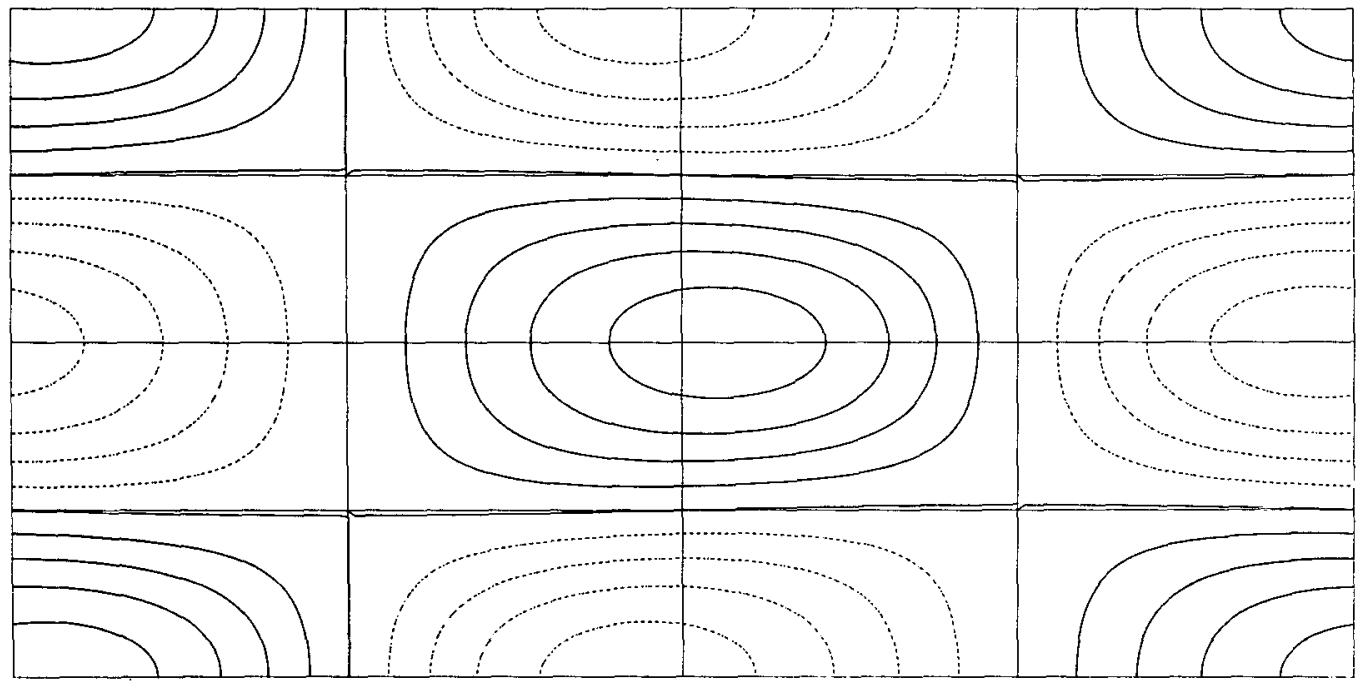

FIG. 3. (Continued)

The balance equation relating geopotential and streamfunction derived from (9) to (11) is

$$
\nabla^{2} \phi^{\prime}=\left(f_{0}+\beta y\right) \nabla^{2} \psi^{\prime}+\beta \partial \psi^{\prime} / \partial y,
$$

which is a form of the linear balance equation retaining the variation of Coriolis parameter $\left[\nabla^{2} \phi=\nabla \cdot(f \nabla \psi)\right]$. In the usual quasi-geostrophic analysis this would be approximated by neglecting terms involving $\beta$, giving the geostrophic streamfunction $\psi^{\prime}=\phi^{\prime} / f_{0}$. However, this neglects the ageostrophic motion that arises from variations in the Coriolis parameter. The solution for the geopotential, given in Appendix B, is most readily found by inspection of the momentum equations (9) and (10) after substitution of the solution for $\psi^{\prime}$.

Consider first partitioning the perturbation flow into geostrophic and ageostrophic components based on type-0 geostrophy $\left(f_{0} \mathbf{V}_{g 0}^{\prime}=\mathbf{k} \times \nabla \phi^{\prime}\right)$. The ageostrophic0 winds are shown, together with the perturbation geopotential in Fig. 3a, and with the total geopotential in Fig. 3b.

Since the geostrophic-0 flow is nondivergent, the actual divergence is correctly given by the ageostrophic0 divergence, which in this case is everywhere zero. The interpretation of the ageostrophic- 0 motion is made clear by writing the momentum equations in vector form as

$$
(\partial / \partial t+\bar{u} \partial / \partial x) \mathbf{V}^{\prime}=-f\left(\mathbf{k} \times \mathbf{V}_{a 0}^{\prime}\right)-\beta y\left(\mathbf{k} \times \mathbf{V}_{g 0}^{\prime}\right) .
$$

The first term on the right-hand side gives an acceleration simply by rotating the ageostrophic- 0 wind vector clockwise by $\pi / 2$ (Northern Hemisphere).
However, the second term tends to cancel this, increasingly away from the central latitude $y=0$, so it is not possible to visualize the accelerations from the ageostrophic-0 flow alone. From Fig. 3a the strongest meridional ageostrophic- 0 flow is not in the jet entrance and exit regions, but rather where the meridional geostrophic-0 flow is greatest. There is therefore a great deal of cancellation between the two Coriolis terms in (13).

The basic state obeys $f \bar{u}=-\partial \bar{\phi} / \partial y$, but geostrophy0 defines $f_{0} \bar{u}_{g o}=-\partial \bar{\phi} / \partial y$, so an "ageostrophic" component is also needed, obeying $f \bar{u}_{a 0}=-\beta y \bar{u}$. This becomes important in the total KE equation. The kinetic energy is $k=\frac{1}{2}\left(u^{2}+v^{2}\right)=\frac{1}{2} \bar{u}^{2}+\bar{u} u^{\prime}+\frac{1}{2}\left(u^{\prime}+v^{\prime 2}\right)$ and is made up of the KEs of the basic state and perturbation flows plus a contribution which depends on both, the "correlation" $\mathrm{KE}$. The latter dominates in the variation of total $\mathrm{KE}$, the equation for which becomes

$(\partial / \partial t+\bar{u} \partial / \partial x) k=-V_{a 0}^{\prime} \cdot \nabla \phi^{\prime}-\bar{u}_{a 0} \partial \phi^{\prime} / \partial x-v_{a 0}^{\prime} \partial \bar{\phi} / \partial y$.

This states that the KE generation rate provides the change in $\mathrm{KE}$ following the basic state flow. However, there is a contribution from $\bar{u}_{a 0}$ which tends to cancel that from $v_{a 0}^{\prime} \partial \bar{\phi} / \partial y$ away from the central latitude. Thus the total generation rate of KE shown in Fig. $3 c$ depicts the jet entrance and exit accelerations, whereas the perturbation ageostrophic- 0 wind in Fig. $3 \mathrm{~b}$ is largest elsewhere.

Consider now geostrophic partitioning of the flow based on type-1 geostrophy. The geostrophic-1 wind is now $f \mathbf{V}_{g 1}^{\prime}=\mathrm{k} \times \nabla \phi^{\prime}$, which has divergence $D_{g 1}$ $=-\beta v_{g 1}^{\prime} / f$. The resulting ageostrophic-1 wind field is 
shown, together with the perturbation geopotential in Fig. 4a, and with the total geopotential in Fig. 4b.

In contrast to the ageostrophic-0 flow in Fig. 3, the ageostrophic- 1 flow is now strongly divergent. However, the divergences of the ageostrophic-1 and geostrophic-1 flows cancel everywhere, since the total flow is nondivergent. Thus it would be entirely erroneous to infer any vertical motion from the ageostrophic-1 flow. Interpretation of the ageostrophic- 1 flow is simpler than for the ageostrophic-0 flow since the momentum equation can now be written

$$
(\partial / \partial t+\bar{u} \partial / \partial x) \mathbf{V}^{\prime}=-f\left(\mathbf{k} \times \mathbf{V}_{a 1}^{\prime}\right)
$$

The acceleration following the basic state flow is given

(a) PERTURBATION GEOPOTENTIAL

AGEOSTROPHIC-1 WIND CONTOUR INTERVAL
50 JKG $\stackrel{\text { ARROW SCAL ING }}{\longrightarrow} 0.25 \mathrm{M} / \mathrm{S}$



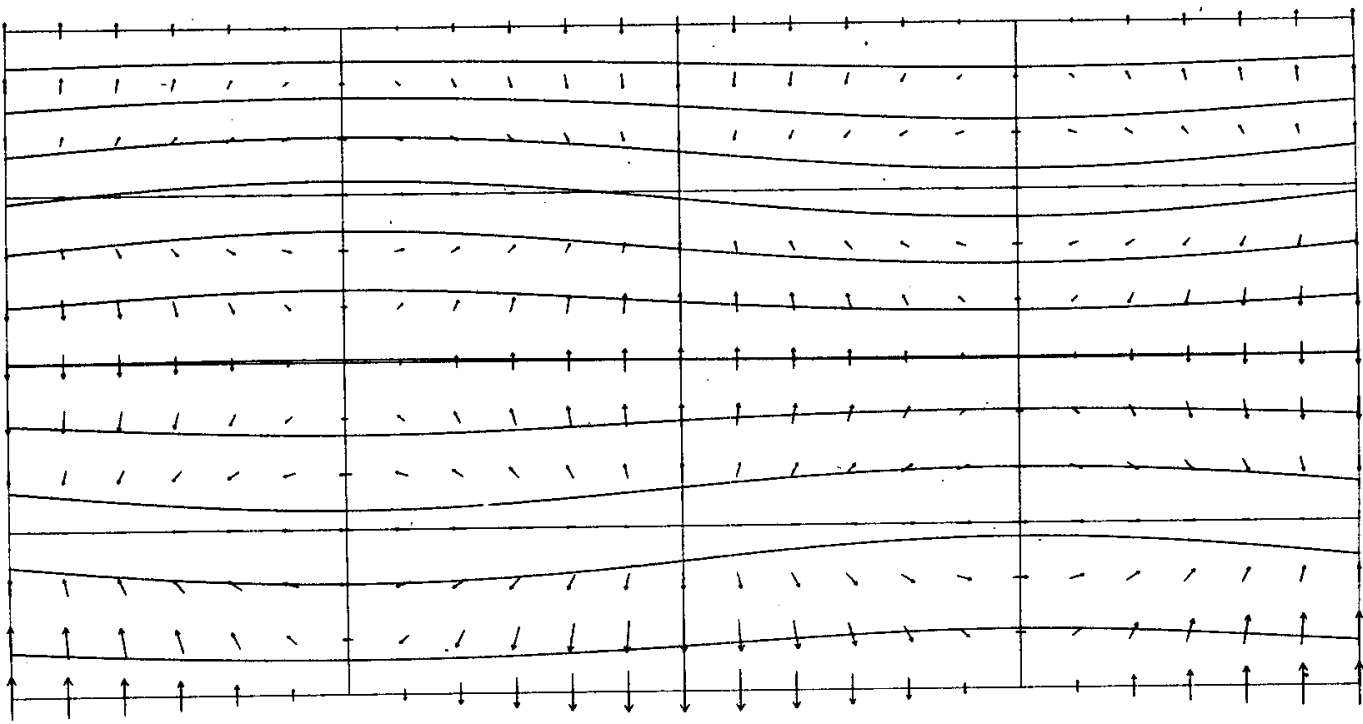

FIG. 4. Ageostrophic-1 winds for the Rosshy mode as in Fig. 3, but based on the variable Coriolis parameter type of geostrophy and scaled up by a factor of 2 . The ageostrophic- 1 flow is now completely divergent, cancelling the geostrophic-1 divergence everywhere. The KE generation rate is as in Fig. 3, but now the accelerations can be visualized geostrophic-1 divergence everywher resemblance to that observed in the climatological jetstreams (see Fig. 8a), yet is associated with nondivergent flow. 
(a)



(b)



(c)

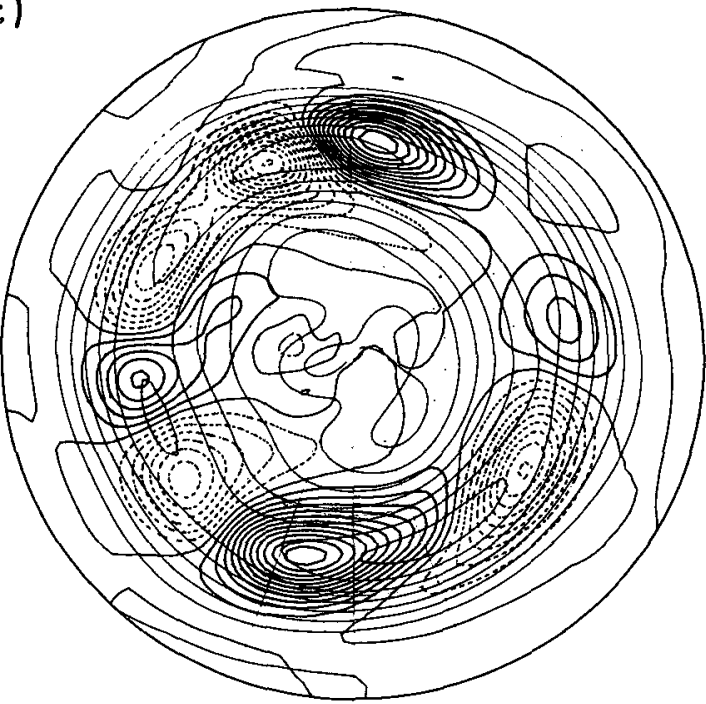

(d)



(e)

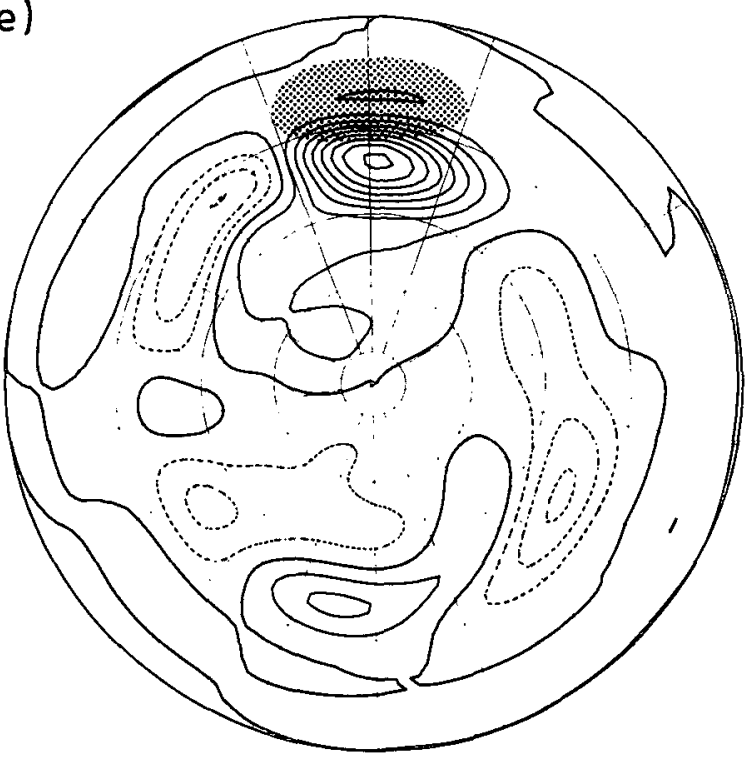

Fig. 5. Fields at sigma $=0.3(300 \mathrm{mb})$ for Hoskins and Karoly's (1981) steady-state linear solution to localized deep heating in the subtropics. The projection is polar stereographic. (a) Perturbation streamfunction, contour interval $10^{6} \mathrm{~m}^{2} \mathrm{~s}^{-1}$, showing the tropical response and propagating Rossby wavetrain. (b) Divergence, contour interval $10^{-7} \mathrm{~s}^{-1}$. The heating region coincides with the region of strong divergence at $15^{\circ}$ latitude. (c) Isotachs (faint lines) and $\mathrm{KE}$ generation rate (bold lines), contour intervals $5 \mathrm{~ms}^{-1}$ and $2.5 \times 10^{-4}$ $\mathrm{W} \mathrm{kg}{ }^{-1}$, showing localized jets in the basic-state westerly flow. Contributions to the generation rate from (d) rotational, and (e) divergent winds, contour interval $2.5 \times 10^{-4} \mathrm{~W} \mathrm{~kg}^{-1}$. The heating region is denoted in (d) and (e) by shading, as in Hoskins and Karoly (1981, Fig. 3c). Negative contours are dashed throughout. KE generation and therefore ageostrophic flow is dominated, particularly in the Rossby wavetrain, by the rotational flow. 
entirely by the ageostrophic-1 wind and can be visualized by rotating the ageostrophic-1 wind vector clockwise through $\pi / 2$ in the Northern Hemisphere. It can be seen from Fig. 4 that the zonal accelerations are provided by $v_{a 1}^{\prime}$ as air passes eastwards through the jet regions, while meridional accelerations are provided by subgeostrophic flow in the troughs and supergeostrophic flow in the ridges.

The basic state still obeys $f \bar{u}=-\partial \bar{\phi} / \partial y$, but this is satisfied by $\bar{u}_{g 1}=\bar{u}$ because of the variable $f$ definition of geostrophy-1. The total KE is defined as before, but its balance is now

$$
(\partial / \partial t+\vec{u} \partial / \partial x) k=-\mathbf{V}_{a 1}^{\prime} \cdot \nabla \phi^{\prime}-v_{a 1}^{\prime} \partial \bar{\phi} / \partial y
$$

The balance is unchanged physically, and the total generation rate is as depicted in Fig. 3c. However, the $\mathrm{KE}$ generation is visualized much more readily in terms of the perturbation ageostrophic-1 flow, and the sources and sinks in the jet entrance and exit are given simply by the meridional ageostrophic-1 flow up and down the basic state gradient of geopotential.

This analysis of a nondivergent Rossby mode verifies in a well-known situation that the nature and role of the ageostrophic flow depend crucially on the choice of type of geostrophy. With geostrophy-0, the ageostrophic- 0 divergence correctly yields the vertical motion (or lack of it in this case), but there is no simple relationship between the ageostrophic- 0 wind and accelerations. In contrast, geostrophy-1 results in an ageostrophic-1 divergence that bears no relationship, at least here, to the actual vertical motion, though accelerations of air passing through the retrogressing wave are readily visualized in terms of the ageostrophic-1 wind vectors. Despite the ageostrophic- 1 flow being highly divergent, the total wind is nondivergent, so all $\mathrm{KE}$ generation is associated with the rotational flow through $-V_{\psi} \cdot \nabla \phi$. Air parcels merely slide up and down isobaric surfaces in closed circulations, converting their energy between gravitational potential energy and $\mathrm{KE}$.

The ageostrophic-1 flow in Fig. $4 \mathrm{~b}$ bears some resemblance to that observed in the climatological jet streams in that the meridional component is in the same sense as that observed and is highly divergent (the zonal component is in the opposite sense to that observed). However, the ageostrophic-1 signature in Fig. $4 \mathrm{~b}$ is no more than a kinematic requirement of a horizontally nondivergent retrogressing wave pattern, suggesting that at least part of the observed ageostrophic- 1 flow in the atmosphere may be associated with purely rotational flow rather than vertical meridional circulations. Since the wavelike solutions of (9)(11) are also solutions of the nonlinear barotropic vorticity equation, obeying the same dispersion relation, all unforced pure waves in horizontally nondivergent flow must exhibit the same behavior.

\section{Analysis of a dissipative model system}

The energetic signatures of the previous section can only apply to systems in which there is no frictional dissipation of KE. When dissipation is present there must be a net throughput of energy from the forcing mechanism involving, in the case of heating, a net conversion from thermal energy to KE. This requires divergent circulations of the type shown in Fig. 1. However, it is possible that the local energetics are still dominated by the "neutral" rotational component that is characteristic of free horizontally nondivergent modes.

In this section, therefore, a dissipative model situation is considered that involves thermal forcing. It is the steady-state linear response to localized deep heating in the subtropics described by Hoskins and Karoly (1981). This solution has no transients but includes biharmonic $\nabla^{4}$-type horizontal diffusion of vorticity, divergence and temperature plus linear damping to parameterize a boundary layer, radiative and transient effects (see Hoskins and Karoly, 1981, for details). The
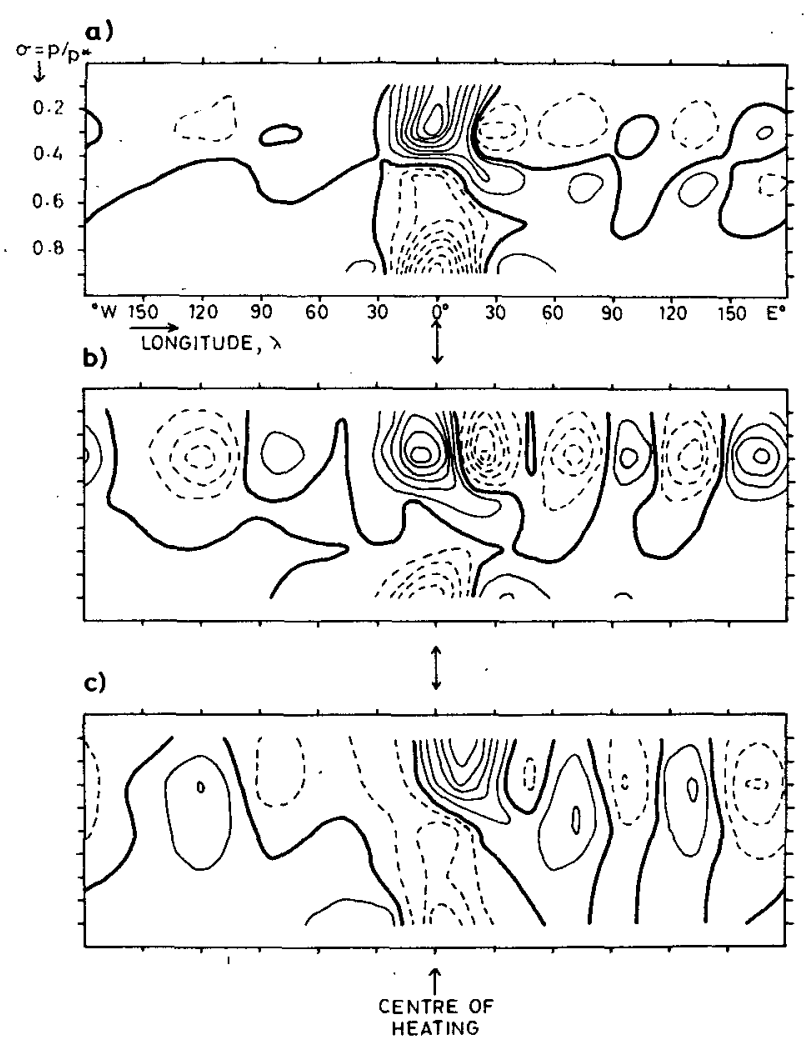

Fig. 6. Vertical cross sections of (a) divergence and its (b) ageostrophic-1 and (c) geostrophic-1 components along $14^{\circ} \mathrm{N}$ in Hoskins and Karoly's model solution. Contour interval is $2.5 \times 10^{-7} \mathrm{~s}^{-1}$ with negative values dashed. Note the tendency for cancellation between the components so that, at upper levels, the ageostrophic-i divergence greatly overestimates the actual vertical motion. 
basic-state zonal flow is a December-January-February climatology.

The model response may be split into two parts, each of which has localized jet streams associated with it:

1) A local tropical response which may be described as a westward propagating internal Rossby mode, giving a localized westerly jet stream, poleward of the heating region.

2) An "equivalent barotropic" Rossby wavetrain which propagates poleward and eastward away from the forcing region. There are localized jets where this crosses the basic-state westerly jet.

\section{a. Local response}

As noted by Hoskins and Karoly, the heating is balanced locally by ascent and the vorticity equation is dominated by the Sverdrup balance, as in Gill's (1980) model. At upper levels there is an anticyclone to the west, with equatorward flow through the divergent region, as can be seen from the perturbation streamfunction and divergence at $300 \mathrm{mb}(\sigma=0.3)$ in Figs. $5 \mathrm{a}$ and $5 \mathrm{~b}$. Figure $5 \mathrm{c}$ shows the isotachs and $\mathrm{KE}$ gen- eration rate at the same level. The basic westerly jet has been strengthened locally, poleward and slightly downstream of the heating region, with a jet entrance region $10-20^{\circ}$ upstream and exit from about $30-60^{\circ}$ downstream. The divergence field in Fig. 5 b shows the descent to be concentrated to the north of the jet entrance and also further west, so there is a thermally direct meridional circulation there, consistent with Blackmon et al.'s (1977) model.

However, the main KE generation, and therefore ageostrophic-1 flow, is west of the main divergent flow as can be seen from Figs. 5d and 5e, which show the contributions to the generation rate from the rotational and divergent winds. The rotational-wind contribution is approximately longitudinally symmetric about the heating region, tending to move the main jet entrance to the west of that given by the divergent wind alone. Thus the relationship between the jet entrance region and the tropical heating is complicated beyond Blackmon et al.'s model by the fact that the rotational wind also contributes to KE generation. This is more marked at $100 \mathrm{mb}$ (not shown), where the jet center is located directly poleward of the heating.

(a)

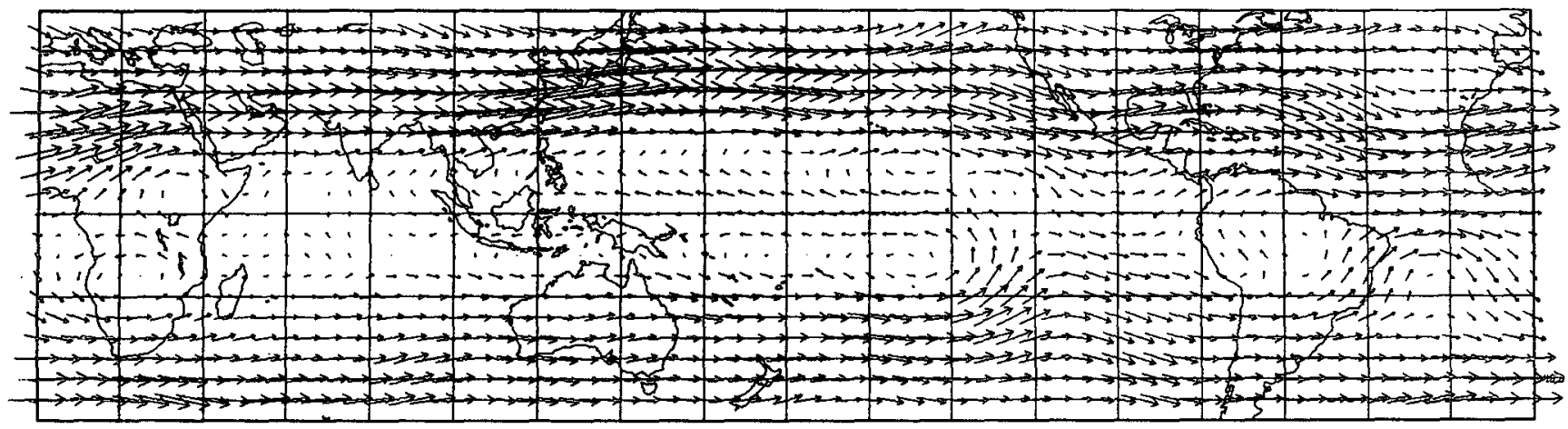

(b)



FIG. 7. Time averaged flow for December-February 1982/83, from ECMWF initialized analyses at $1200 \mathrm{GMT}$. Wind vectors at (a) $150 \mathrm{mb}$ and (b) $850 \mathrm{mb}$, together with the vertical motion (omega $=D p / D t$ ) at $500 \mathrm{mb}$ in $(\mathrm{b})$, contour interval $1.1 \times 10^{-2} \mathrm{~Pa} \mathrm{~s}^{-1}$. Dashed lines denote ascent. The latter has been spectrally filtered to emphasize large-scale features (see text for details). 

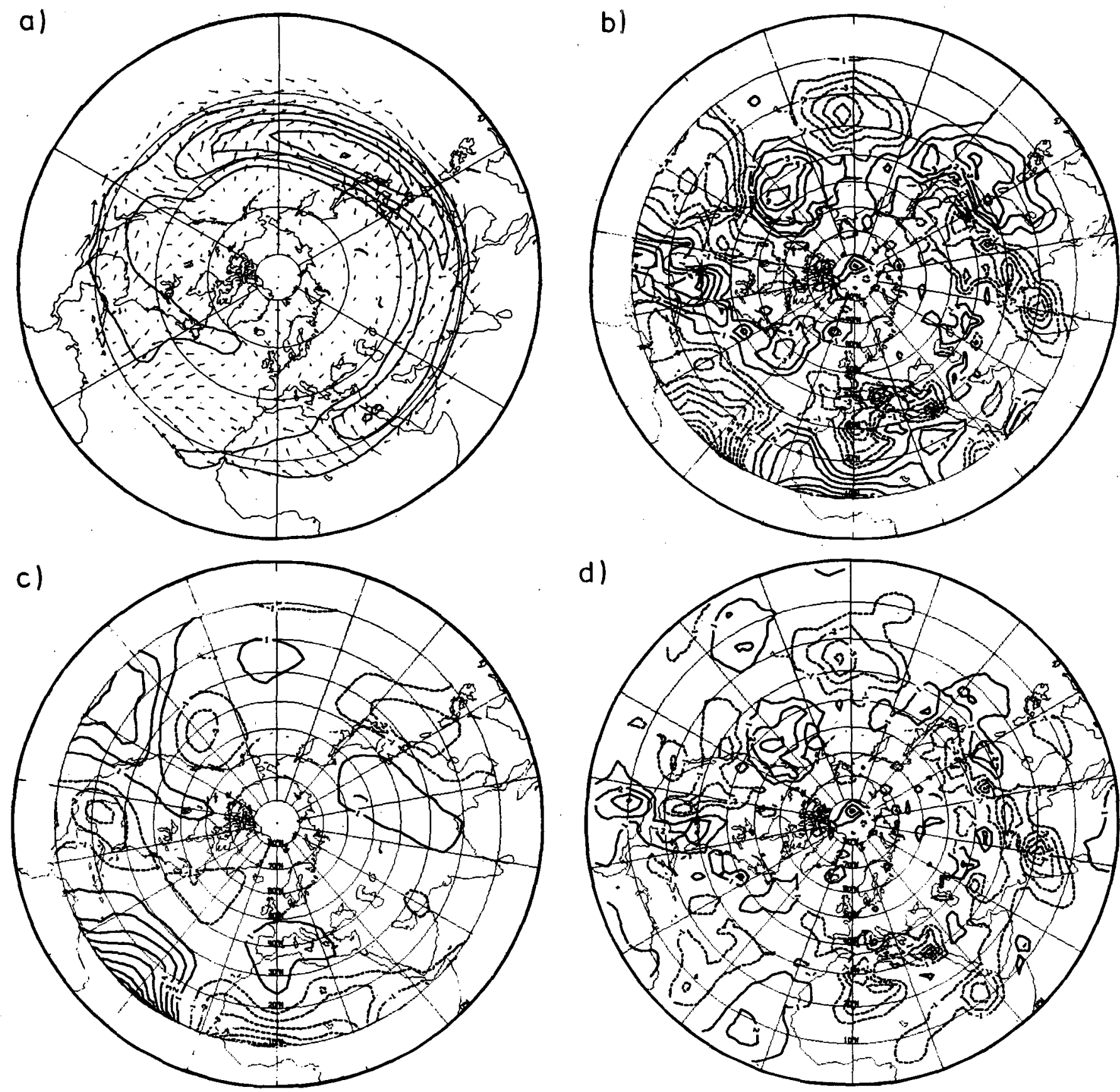

\section{b. Rossby wavetrain}

The isotachs at $300 \mathrm{mb}$ in Fig. 5c show that localized jets are also formed where the Rossby wavetrain crosses the latitude of the basic-state westerly jet, and that there is $\mathrm{KE}$ generation and thus ageostrophic flow in these jet entrances and exits. The clearest example is the jet some $200^{\circ}$ downstream of the heating region.

Comparison with the divergence distribution in Fig. $5 \mathrm{~b}$ reveals there to be thermally direct and indirect meridional circulations in the jet entrances and exits, re-

spectively. However, Fig. 6, which shows vertical sections of the divergence and its geostrophic- 1 and ageostrophic- 1 components along the tropical side of the jets, reveals much cancellation of the two components at $300 \mathrm{mb}$. The total divergence has the same sense and distribution as its ageostrophic-1 component but its magnitude is much reduced because of the opposite sense of the geostrophic-1 component (except in the heating region itself). Note that below this, at 500 and $700 \mathrm{mb}$, the divergence generally has the opposite sign to its ageostrophic-1 component. Thus a 



FIG. 8. Time averaged fields at $250 \mathrm{mb}$ for DJF $1982 / 83$ using a polar stereographic projection. (a) Isotachs (contour interval $10 \mathrm{~m}$ $\mathrm{s}^{-1}$ from $30 \mathrm{~m} \mathrm{~s}^{-1}$ only) and ageostrophic-1 wind vectors. Divergence of the (b) ageostrophic-1, (c) geostrophic-1 and (d) total wind, contour interval $10^{-6} \mathrm{~s}^{-1}$. The ageostrophic-1 divergence substantially overestimates the actual divergence equatorward of about $30^{\circ} \mathrm{N}$. KE generation rate associated with the (e) total wind $-V \cdot \nabla \phi$, (f) rotational wind $-V_{\psi} \cdot \nabla \phi$ and (g) divergent wind $-V_{x} \cdot \nabla \phi$, contour interval $2.75 \times 10^{-3} \mathrm{~W} \mathrm{~kg}^{-1}$. In (b)-(g) positive contours are solid, negative dashed and the zero omitted for clarity. Plots (e)-(g) have been smoothed as in Fig. $7 \mathrm{~b}$.

map of ageostrophic-1 wind vectors would, at upper levels, greatly overestimate the divergence, while at lower levels it would imply the wrong sign. This compares with the nondivergent Rossby mode in which the two divergence components cancelled exactly. Some cancellation is inevitable in an equivalent barotropic structure, since $v_{g 1}$ is in the same sense throughout each vertical column, so that the geostrophic-1 divergence does not integrate to give mass balance. The integral of the ageostrophic- 1 divergence must cancel this to leave, in this case, a simple internal-mode structure to the total divergence. Allied to this, the rotational and divergent wind contributions to the KE generation in Figs. 5d and 5e show that the "neutral" rotational part dominates locally even in this divergent flow. Both components are in the same sense, so the divergent circulation has the structure that would be implied by the ageostrophic- 1 wind vectors, but is of much reduced intensity, consistent with the cancellation between the divergence components at upper levels. 




b)



c)

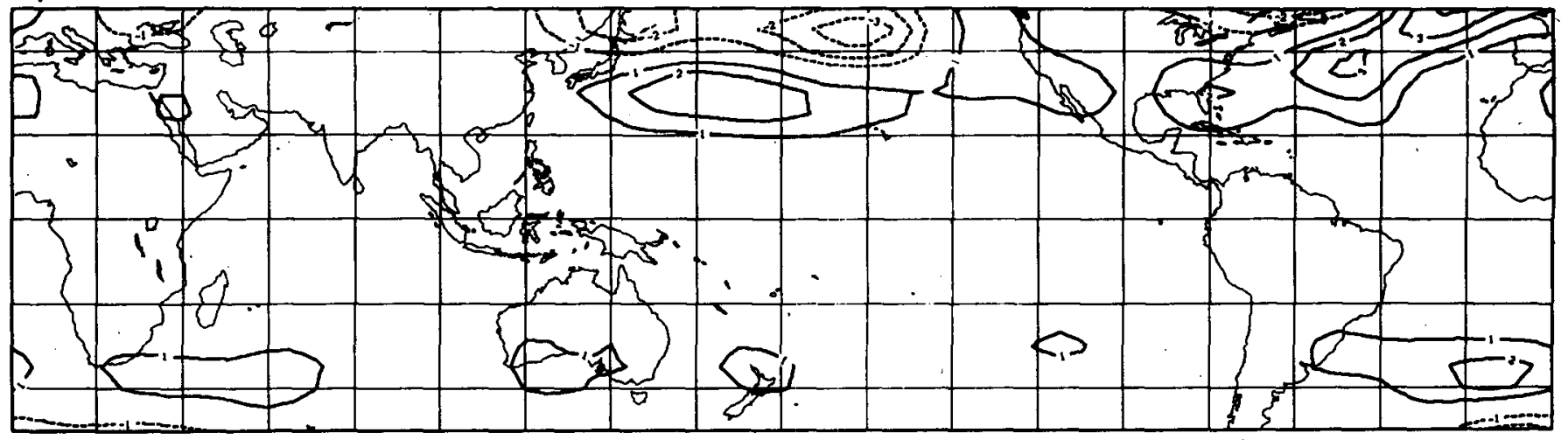

d)

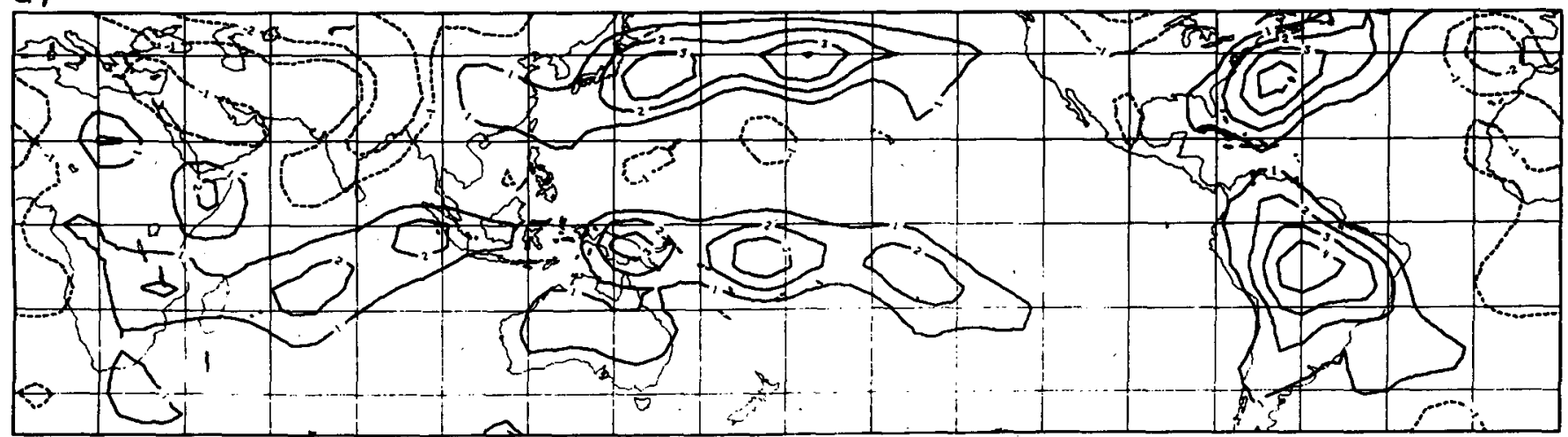


In contrast to the locally forced response, the vertical motion in the wavetrain is very much a second-order effect, necessary to maintain thermal wind balance and satisfy the thermodynamic equation in the baroclinic atmosphere. It is predicted by quasi-geostrophic theory (Hoskins et al., 1978) and is the basis of the confluence theory of Namias and Clapp (1949), also used by Blackmon et al. (1977) to infer the presence of meridional circulations around the climatological jet streams. In the absence of a basic state meridional temperature gradient, the nondivergent Rossby mode kinematics of the previous section would prevail. However, the meridional motion involved in such a response gives rise to thermal advection in a baroclinic atmosphere, which must be balanced by adiabatic warming and cooling associated with vertical motion.

It must be concluded that the "neutral" rotational component is dominating the local energetics at the jet stream level in the Rossby wavetrain. The kinematics of wave retrogression are still largely accomplished by rotational flow, while the divergent flow makes a smaller local contribution though, in the isobaric average, it is this component alone that must balance frictional dissipation.

\section{Northern Hemisphere wintertime circulation}

In this section the Northern Hemisphere wintertime circulation is studied using data from the initialized analyses at the European Centre for Medium Range Weather Forecasts (ECMWF). The 1200 GMT analyses have been used to obtain the time-averaged flow for the period December-January-February 1982-83. The ageostrophic-1 flow will be analysed at $250 \mathrm{mb}$, which is a compromise between the level of jet stream maxima in midlatitudes and the main level of tropical divergent outflow around $150 \mathrm{mb}$.

The major features of the divergent part of the tropical circulation should be present in the data since ECMWFs nonlinear normal mode initialization has included diabatic processes since September 1982. Sardeshmukh and Hoskins (1985) discuss the vorticity balance at $150 \mathrm{mb}$ for the period studied here using the same data. They find evidence that the pattern of tropical divergence is correctly captured, but that its intensity is slightly underestimated. Their success in using a nonlinear vorticity equation balance to obtain the seasonal mean rotational flow diagnostically from the divergent flow lends further credence to the latter in the ECMWF data.

The main features of the global circulation are evident from Fig. 7, depicting the time-averaged flow at
150 and $850 \mathrm{mb}$, together with the vertical motion at $500 \mathrm{mb}$. The latter has been filtered with a spectral filter of the form $s_{n}=\exp \left\{-K[n(n+1)]^{2}\right\}$ for $n \leqslant 24$, $s_{24}=0.1$, where $n$ is the total wavenumber on the sphere (see Sardeshmukh and Hoskins, 1984), to emphasize the coherent large-scale features.

The major northern Pacific jet stream is approximately in its climatological position off the Asian coast, though extending farther eastward than normal, with weaker jets over the Atlantic and Middle East. This winter was characterized by a strong El Niño and the tropical circulation was highly anomalous, the main heating region being displaced east of its climatological position over Indonesia into the mid-Pacific. The main tropical ascent given by the $500 \mathrm{mb}$ omega field extends eastwards from about $140^{\circ} \mathrm{E}$, and there are upper-level easterlies across much of the tropical Pacific. Also of note are the tightly confined vertical circulations in the entrance $\left(100-150^{\circ} \mathrm{E}\right)$ and exit $\left(170^{\circ} \mathrm{E}-120^{\circ} \mathrm{W}\right)$ of the Pacific jet, consistent with Blackmon et al.'s model.

The time-mean isotachs and ageostrophic- 1 flow at 250 mb shown in Fig. 8a reveal ageostrophic-1 circulations in the jet entrance and exit regions in the sense required to provide the acceleration of air passing eastwards through them. Both the ageostrophic-1 vectors in Fig. 8a and their divergence in Fig. 8b show these circulations to be highly divergent, but this is partially cancelled by the geostrophic-1 divergence (Fig. 8c) to leave a generally weaker distribution of the total horizontal divergence (Fig. 8d). Equatorward of about $25^{\circ}$, there is almost complete cancellation, particularly where there is strong meridional motion (e.g., $120^{\circ} \mathrm{W}$, $90^{\circ} \mathrm{W}, 50^{\circ} \mathrm{W}$ and over North Africa), so that the total divergence is not well correlated with its ageostrophic1 component. However, in midlatitudes, the ageostrophic-1 divergence dominates so that the total divergence has the same sense but is weaker. Only in the Pacific jet exit $\left(170^{\circ} \mathrm{E}\right.$ to $\left.120^{\circ} \mathrm{W}\right)$ is the vertical circulation accurately implied by the ageostrophic-1 divergence. The jet entrance circulation (centered on $120^{\circ} \mathrm{E}$ ) and those associated with the Atlantic jet are much weaker, while there is descent rather than ascent on the equatorward side of the jet entrance over North Africa (Figs. $7 \mathrm{~b}$ and $8 \mathrm{~d}$ ).

The fact that the total divergence is weaker than that of the ageostrophic-1 flow implies that accelerations in the jet entrances and exits are at least partially associated with rotational flow. The degree to which this is true is dramatically revealed in Figs. 8e-g, which show the KE generation rate associated with the total wind and its rotational and divergent components, respectively. As in the model situation of the preceding

FIG. 9. Terms in the time averaged and vertically integrated heat balance for DJF 1982/83. All have been smoothed as in Fig. $7 \mathrm{~b}$. Cooling associated with (a) time averaged horizontal advection, (b) time averaged vertical advection and (c) transient fluxes. (d) is the diabatic heating rate calculated as a sum of (a), (b) and (c). Contour interval is $44 \mathrm{~W} \mathrm{~m}^{-2}$. The balance in the jet entrance and exit circulations is between the time averaged horizontal and vertical advection, indicating that these circulations are geostrophically rather than diabatically forced. 
section, purely nondivergent processes dominate, with the contribution from the divergent wind being generally in the same sense as the total, but several times smaller.

The vertical circulations which are present in the jet entrances and exits are tightly confined around the jet axis. The circulation in the Pacific jet entrance has its ascent at $20-30^{\circ} \mathrm{N}$ (Figs. $7 \mathrm{~b}$ and $8 \mathrm{~d}$ ), well away from the main region of tropical ascent associated with convection, which is just south of the equator (Fig. 7b). This suggests that the ascending branches of the direct circulations are not in areas of strong convection and latent heating, so differ from the locally-forced jet entrance circulation in Section 4.

The time-averaged thermodynamic equation is sufficient to determine what processes balance the adiabatic warming and cooling in the vertical branches of the jet circulations. Partitioning into time-mean $\left(^{-}\right.$) and transient ()$^{\prime}$ components, the thermodynamic equation (3), including diabatic heating rate $q$, may be written

$$
\begin{aligned}
\overline{\mathbf{v}} \cdot \nabla C_{p} \bar{T}+C_{p}\left(\frac{p}{p_{0}}\right)^{\kappa} \bar{\omega} \frac{\partial \bar{\theta}}{\partial p} \\
+C_{p}\left(\frac{p}{p_{0}}\right)^{\kappa}\left[\nabla \cdot \overline{\mathbf{V}^{\prime} \theta^{\prime}}+\frac{\partial}{\partial p}\left(\overline{\omega^{\prime} \theta^{\prime}}\right)\right]=\bar{q},
\end{aligned}
$$

where $p_{0}=1000 \mathrm{mb}$. The first term is the horizontal advection by the time-mean flow, the second the adiabatic cooling associated with mean vertical motion and the third the effect of transients.

Figure 9 shows the distribution of the four terms of the heat balance, integrated over pressure in the vertical, for the 1982/83 northern winter. The diabatic heating is calculated as the sum of the adiabatic tendencies. The plots have been filtered as in Fig. 7. The dominant balance in the jet entrance and exit regions is between the mean horizontal and vertical advection, particularly for the Pacific jet. In the centers of the Pacific and Atlantic jets, the transients transport heat polewards. Latent heating is also important, so all four terms contribute to the balance there. The horizontal advection in Fig. 9 does not distinguish between its geostrophic- 1 and ageostrophic- 1 components, but the scale analysis of Section 2 implies that the geostrophic1 component dominates. In the time-average, the firstorder thermodynamic equation is sufficient to determine the geostrophically forced vertical motion given the geostrophic-1 flow (see Appendix A), because the local rate of change is zero, so the full $Q$-vector analysis is not necessary. However, the $Q$-vectors (not shown) do also imply adiabatically and geostrophically forced vertical motion around the jets of the same sense and distribution as that in Fig. 9b.

These observations indicate that the vertical circulations associated with climatological jet streams are secondary circulations, and are necessary to maintain thermal balance in the presence of time-mean hori- zontal advection by the geostrophic flow. This is the same signature as was found in the remotely forced Rossby wavetrain in Section 4. Any diabatically forced component of the observed circulations can form only a residual part of the thermal balance.

\section{Conclusions}

The reason for problems of interpretation of ageostrophic flow is the use of two types of geostrophy, one in theoretical studies and the other in studies of atmospheric data. To zero order in a Rossby number expansion the two types of geostrophic flow are equivalent, but the first-order equations and ageostrophic flow have quite different interpretations. For this reason a nomenclature has been suggested to distinguish between them. Type- 0 geostrophic flow uses a constant value of the Coriolis parameter, and so is nondivergent (type- 0 is intended as a mnemonic for this $f_{0}$ definition). In contrast type-1 geostrophy uses the local value of $f$. The geostrophic-1 divergence is not negligible so the ageostrophic-1 flow cannot be used to imply the vertical motion. Only the ageostrophic- 0 flow completely defines the vertical motion.

The importance of the consistent use of a particular diagnostic throughout model and data studies cannot be stressed enough. Apart from the problem of two types of geostrophy, the momentum and energetics analysis involving the ageostrophic flow appears never to have been applied to fundamental conceptual models. It has been applied here to the nondivergent Rossby mode and shows the ageostrophic flow to be fundamental to the wave's retrogression. However, the ageostrophic flow has been found to be a cumbersome diagnostic tool, particularly since the KE balance does not efficiently separate advective and divergent processes. In contrast the vorticity balance provides a superior separation, and forcing of vorticity is related simply to the local divergence. Now that modern data analysis and initialization processes allow the major features of the divergence field to be computed, it appears that the vorticity balance offers a more promising way of analyzing flow structures in atmospheric as well as model data. The thermodynamic equation still provides a link with the diabatic heating, either separately or combined in the balance of the more conservative potential vorticity. The strength of the energetics analysis appears to lie in its global rather than local constraints on the flow.

On the positive side, the momentum and energetics analysis here has shown that ageostrophic flow is largely determined by the pure kinematics of wave retrogression. In a nondivergent Rossby mode the retrogression is achieved entirely by rotational flow. This is still the dominant process in a model equivalent barotropic Rossby wavetrain and in the Northern Hemisphere wintertime jet streams. The vertical circulations that are present in the entrances and exits of the atmospheric jet streams are in the same sense as would be implied 
by the ageostrophic-1 flow alone although weaker. The heat balance has shown these circulations to be primarily geostrophically forced, and so to be secondary circulations necessary to maintain thermal balance.

Acknowledgments. I would like to thank Professors B. J. Hoskins and R. P. Pearce for their support and contribution to this work. The figures in Section 5 were produced as part of a joint diagnostics project between Reading University and the U. K. Meteorological Office using ECMWF data, by Drs. Prashant D. Sardeshmukh and Glenn H. White. Several other figures were drafted by Mrs. K. V. Daykin. The work was supported by a University of Reading studentship, forming part of a doctoral thesis, and later by a NERC research fellowship.

\section{APPENDIX A}

\section{$Q$-Vectors on a $\beta$-Plane}

The diagnosis of vertical motion on an $f$-plane in terms of $Q$-vectors has been discussed by Hoskins et al. (1978, hereafter denoted HDD). In that case, both types of geostrophy are equivalent and a horizontally nondivergent flow forms the zero-order balance. When $f$ varies, on a $\beta$-plane or the sphere, the $Q$-vector formulation can be approached using either type of geostrophy. The main difference between the two is in the circulation equation, which favors the geostrophy-1 formulation despite the vertical motion not being entirely associated with the ageostrophic-1 flow (B. J. Hoskins, personal communication, 1984).

Using the scaling and Rossby number expansion method of Section 2 and a vertical coordinate $z=\left(R \theta_{0} /\right.$ $\left.g_{\kappa}\right)\left[1-\left(p / p_{0}\right)^{\kappa}\right]$ as in HDD, where $\theta_{0}$ and $p_{0}$ are constant values, the first-order momentum equation for geostrophy-1 is (7), while the thermodynamic, hydrostatic and continuity equations become

$$
\begin{aligned}
\left(\partial / \partial t+\mathbf{V}_{g 1} \cdot \nabla\right) \theta & =-w \partial \Theta / \partial z \\
\partial \phi / \partial z & =\left(g / \theta_{0}\right) \theta \\
\nabla \cdot \mathbf{V}_{a 1}+\partial w / \partial z & =\beta v_{g 1} / f,
\end{aligned}
$$

where $w=D z / D t$ is the vertical velocity and $\Theta(z)$ is a standard potential temperature distribution with squared buoyancy frequency $N^{2}=\left(g / \theta_{0}\right) \partial \Theta / \partial z$.

The tendency of geostrophic-1 flow to destroy its thermal wind balance is evident from the equations for the components of the balance with the ageostrophic-1 and vertical motion omitted. The $y$-momentum and thermodynamic equations are differentiated to give

$$
\begin{gathered}
\left(\partial / \partial t+\mathbf{V}_{g 1} \cdot \nabla\right)\left(f \partial v_{g 1} / \partial z\right)=-Q_{1}+2 \beta v_{g 1} \partial v_{g 1} / \partial z \\
\left(\partial / \partial t+\mathbf{V}_{g 1} \cdot \nabla\right)\left(\frac{g}{\theta_{0}} \partial \theta / \partial x\right)=Q_{1}
\end{gathered}
$$

where

$$
\mathbf{Q}=\left(Q_{1}, Q_{2}\right)=-\left(\frac{g}{\theta_{0}}\right)\left[\frac{\partial V_{g 1}}{\partial x} \cdot \nabla \theta, \frac{\partial V_{g 1}}{\partial y} \cdot \nabla \theta\right] .
$$

The scaling assumptions of Section 2 show the extra term $2 \beta v_{g 1} \partial v_{g 1} / \partial z$ to be $\mathrm{O}(2 L / a)$ relative to $Q_{1}$, so it can be neglected to this order. Then the geostrophic-1 flow changes the components of its thermal wind balance by equal and opposite amounts, as in $f$-plane theory (HDD).

Including the ageostrophic- 1 and vertical motion in this analysis, the components of the thermal wind balance can be eliminated to give the circulation equation

$$
N^{2} \nabla w-f_{0}^{2} \partial \mathbf{V}_{a 1} / \partial z=2 \mathbf{Q},
$$

accurate to zero order in Ro. Terms $f_{0} \beta y \partial \mathbf{V}_{a 1} / \partial z$ and $2 \beta v_{g 1} \mathbf{k} \times \partial \mathbf{V}_{g 1} / \partial z$, both $\mathrm{O}(\mathrm{Ro})$ relative to the $Q$-term, have been neglected. This equation defines the geostrophically forced secondary circulation, consisting of the ageostrophic- 1 and vertical motion, necessary to maintain thermal wind balance. Since the neglected terms are $\mathrm{O}(\mathrm{Ro})$ relative to the $Q$-term, the interpretation and sense of direct and indirect forcing in HDD is retained. Using geostrophy-0, the extra term arising in (Al) is $\beta y f_{0} \partial \mathrm{V}_{g 0} / \partial z$, which is of the same order as the $Q$-term. Then the balance away from the $f_{0}$ latitude in (A1) is increasingly dominated by cancellation between the forcing terms-making interpretation more difficult. Thus the geostrophy-1 formulation is preferable.

The omega equation derived from (A1) is

$$
N^{2} \nabla^{2} w+f_{0}^{2} \frac{\partial^{2} w}{\partial z^{2}}=2 \nabla \cdot \mathbf{Q}+f_{0} \beta \frac{\partial v_{g 1}}{\partial z},
$$

where again terms of $O(R o)$ have been neglected. The extra term on the right-hand side is of the same order as the $Q$-vector term, so it cannot be neglected. It is discussed in HDD and arises directly from the divergence of the geostrophic-1 wind in the derivation here. However, in jet entrance and exit regions, where the $x$ and $y$ length scales are related by $L_{x} \sim 3 L_{y}$, the $Q_{2}$ term dominates and is $\mathrm{O}\left(2 L_{x} / L_{y}\right)$ compared with the extra term. Thus the $Q$-vector should still give the geostrophically forced vertical motion in the jet streams. However, the extra term in the omega equation must generally be considered when $f$ varies and the vertical motion will not simply be given by the $Q$ vector.

\section{APPENDIX B}

\section{Nondivergent Rossby Mode Solutions}

Solutions of the vorticity equation (15) are plane waves of the form $\psi^{\prime}=\operatorname{Re}\left\{A e^{i \phi}\right\}$, where $A=(a-i b)$ and $\phi=k x \pm l y-\nu t$, with $k$ and $l$ zonal and meridional wavenumbers and $\nu$ frequency. They obey the dispersion relation $c=\bar{u}-\beta /\left(k^{2}+l^{2}\right)$, where $c=\nu k$ is the zonal phase speed. Adding the two solutions $\phi_{1}=k x$ 
$+l y-\nu t$ and $\phi_{2}=k x-l y-\nu t$ and taking $A=a$ yields a particular solution that may be visualized more readily.

Using geostrophy-0 and letting $K^{2}=k^{2}+l^{2}$, the general solution for the fields is

$$
\begin{aligned}
u^{\prime} & =\operatorname{Re}\left\{\mp i l A e^{i \phi}\right\} \\
v^{\prime} & =\operatorname{Re}\left\{i k A e^{i \phi}\right\} \\
\phi^{\prime} & =\operatorname{Re}\left\{\left[f_{0}+\beta y \pm i l \beta / K^{2}\right] A e^{i \phi}\right\} \\
u_{a 0}^{\prime} & =\operatorname{Re}\left\{\left[ \pm i l \beta y / f_{0}+k^{2} \beta / f_{0} K^{2}\right] A e^{i \phi}\right\} \\
v_{a 0}^{\prime} & =\operatorname{Re}\left\{\left[-i k \beta y / f_{0} \pm k l \beta / f_{0} K^{2}\right] A e^{i \phi}\right\} \\
\bar{\phi}(y) & =\bar{\phi}_{0}-f_{0} \bar{u} y-\frac{1}{2} \beta \vec{u} y^{2} .
\end{aligned}
$$

The corresponding particular solution is (letting $A^{\prime}$ $=2 a)$

$$
\begin{gathered}
\psi^{\prime}=A^{\prime} \cos (k x-\nu t) \cos l y \\
u^{\prime}=A^{\prime} l \cos (k x-\nu t) \sin l y \\
v^{\prime}=-A^{\prime} k \sin (k x-\nu t) \cos l y \\
\phi^{\prime}=A^{\prime} f \cos (k x-\nu t) \cos l y \\
-\left(A^{\prime} l \beta / K^{2}\right) \cos (k x-\nu t) \sin l y \\
u_{a 0}^{\prime}=\left(A^{\prime} \beta / f_{0}\right) \cos (k x-\nu t)\left[\left(k^{2} / K^{2}\right) \cos l y-l y \sin l y\right] \\
v_{a 0}^{\prime}=\left(A^{\prime} \beta / f_{0}\right) \sin (k x-\nu t)\left[-\left(k l / K^{2}\right) \sin l y+k y \cos l y\right] .
\end{gathered}
$$

The solutions for the KE and its generation rate are derived from these functions.

Using geostrophy-1, only the ageostrophic wind components are altered, the general solution being

$$
\begin{aligned}
& u_{a 1}^{\prime}=\operatorname{Re}\left\{\left[k^{2} \beta / K^{2} f\right] A e^{i \phi}\right\} \\
& v_{a 1}^{\prime}=\operatorname{Re}\left\{\left[ \pm k l \beta / K^{2} f\right] A e^{i \phi}\right\}
\end{aligned}
$$

and the particular solution

$$
\begin{aligned}
& u_{a 1}^{\prime}=\left(A^{\prime} k^{2} \beta / f K^{2}\right) \cos (k x-\nu t) \cos l y \\
& v_{a 1}^{\prime}=-\left(A^{\prime} k l \beta / f K^{2}\right) \sin (k x-\nu t) \sin l y .
\end{aligned}
$$

Figures 2 to 4 have been drawn using $\bar{u}=10 \mathrm{~ms}^{-1}$ and an amplitude of $2 \mathrm{~ms}^{-1}$ for $u^{\prime}$ for the stationary wave at $45^{\circ}$ latitude, with zonal wavelength double its meridional wavelength.

\section{REFERENCES}

Blackmon, M. L., J. M. Wallace, N.-C. Lau and S. L. Mullen, 1977: An observational study of the Northern Hemisphere wintertime circulation. J. Atmos. Sci., 34, 1040-1053.

Eliassen, A., 1984: Geostrophy. Quart. J. Roy. Meteor. Soc., 110, 112.

Gill, A. E., 1980: Some simple solutions for heat-induced tropical circulations. Quart. J. Roy. Meteor. Soc., 106, 447-462.

Hoskins, B. J., and D. J. Karoly, 1981: The steady linear response of a spherical atmosphere to thermal and orographic forcing. $J$. Atmos. Sci., 38, 1179-1196.

- 1. Draghici and H. C. Davies, 1978: A new look at the $\omega$ equation. Quart. J. Roy. Meteor. Soc., 104, 31-38.

-_, I. N. James and G. H. White, 1983: The shape, propagation and mean-flow interaction of large-scale weather systems. $J$. Atmos. Sci., 40, 1595-1612.

Namias, J., and P. F. Clapp, 1949: Confluence theory of high tropospheric jet stream. J. Meteor., 6, 330-336.

Pearce, R. P., 1974: The design and interpretation of diagnostic studies of synoptic-scale atmospheric systems. Quart. J. Roy. Meteor. Soc., 100, 265-285.

Phillips, N. A., 1963: Geostrophic motion. Rev. Geophys., 1, 123176.

Rossby, C.-G., and Collaborators, 1939: Relation between variations in the intensity of the zonal circulation of the atmosphere and the displacements of the semi-permanent centres of action. $J$. Mar. Res., 2, 38-55.

Sardeshmukh, P. D., and B. J. Hoskins, 1984: Spatial smoothing on the sphere. Mon. Wea. Rev., 112, 2524-2529.

$\ldots$, and $\ldots, 1985$ : Vorticity balances in the tropics during the 1982-83 El Niño-Southern Oscillation event. Quart. J. Roy. Meteor. Soc., 111, 261-278. 